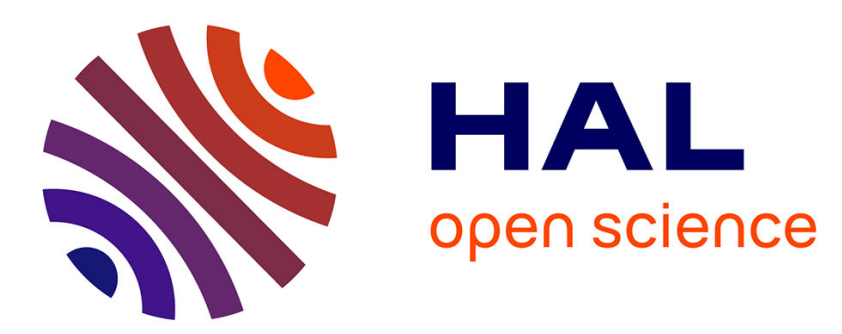

\title{
Derivative-Based Sampled Data Control for Continuous Linear Parameter Varying System With Unknown Parameters
}

Sonia Maalej, Alexandre Kruszewski, Lotfi Belkoura

\section{- To cite this version:}

Sonia Maalej, Alexandre Kruszewski, Lotfi Belkoura. Derivative-Based Sampled Data Control for Continuous Linear Parameter Varying System With Unknown Parameters. Journal of Dynamic Systems, Measurement, and Control, 2019, 141 (8), pp.081013. 10.1115/1.4042947 . hal-02403902

\section{HAL Id: hal-02403902 \\ https://hal.science/hal-02403902}

Submitted on 1 Apr 2020

HAL is a multi-disciplinary open access archive for the deposit and dissemination of scientific research documents, whether they are published or not. The documents may come from teaching and research institutions in France or abroad, or from public or private research centers.
L'archive ouverte pluridisciplinaire HAL, est destinée au dépôt et à la diffusion de documents scientifiques de niveau recherche, publiés ou non, émanant des établissements d'enseignement et de recherche français ou étrangers, des laboratoires publics ou privés. 


\title{
Derivative Based Sampled Data Control For Continuous LPV System With Unknown Parameters
}

\author{
Sonia MAALEJ \\ Laboratory CRIStAL \\ UMR CNRS 9189 \\ Université de Lille 1 \\ BP 48, 59651 \\ Laboratory Non-A, INRIA \\ Email: sonia.maalej@gmail.com
}

\author{
Alexandre KRUSZEWSKI \\ Centrale Lille \\ UMR 9189-CRISTAL \\ Centre de Recherche en Informatique \\ Signal et Automatique de Lille \\ F-59000 Lille, France \\ Email: alexandre.kruszewski@ec-lille.fr
}

\author{
Lotfi BELKOURA \\ Laboratory Non-A, INRIA \\ Cité Scientifique \\ Lille-Nord Europe, France
}

\begin{abstract}
This paper deals with the robust stabilization of a class of Linear Parameter Varying (LPV) systems in the sampled data control case. Instead of using a state observer or searching for a dynamic output feedback, the considered controller is based on output derivatives estimation. This allows the stabilization of the plant with very large parameter variations or uncertainties. The proof of stability is based on the polytopic representation of the closed loop under Lyapunov conditions and system transformations. The result is a control structure with only one parameter tuned via very simple conditions. Finally, the effectiveness of the proposed method is verified via a numerical example of a second order LPV system.
\end{abstract}

\section{Introduction}

In the industrial process control field, most users are using traditional PID controller which was developed in 1940s or improved PID controller. These controllers are very famous because of their low number of tuning parameters and the fact that modeling the plant is not mandatory. Thus, there are often tuned with a simple (linear) non physical model $[1,2]$ leading to poor results when a process has a large operating domain. In addition, due to the non-linear nature of most systems or the variation of their parameters, it is difficult to tune them correctly without a good model or a more complex control structure.

Because internal states of most industrial plants cannot be directly measured and only their outputs are available for control purposes, output feedback design, static output feedback $[3,4]$, dynamic output feedback [5] and fuzzy observer-based control approaches $[6,7]$ have been employed. The design procedure of these controllers needs a good model and for some of them the use of complex optimization techniques. Yet, the choice of the physical model structure, the identification of its parameters and then the experimental validation of it are time-consuming and never simple. Even if the model is available and good enough, using a dynamic model for computing the control law implies the identification of the model parameters.

An important aspect to be considered in the output feedback control problem is the non availability of all variable parameters in real-time for the implementation of the control law. As a matter of fact, the control law depends on the output of the system and on some of the varying parameters. If no information about these variable parameters is available, a constant output feedback control gain may be an alternative but yielding, in general, conservative results.

A solution to those problems is proposed based on some new results in the framework of model free control introduced by Cedric et al. [8]. This framework is signal based and thus don't require many specifications about the system. The main idea of this control law is to nullify the system dynamic and to replace it with the ideal closed loop one. To implement this controller, the output derivatives need to be available. In the literature, many variant of such controller have been developed (see [8-11]). All these 
studies state that the stability as well as performances are ensured but none of them provides a standard proof on a class of system. Recently, the authors of $[12,13]$ have considered the use of the model-free theory and the polytopic transformation to tackle problems related to the stability tuning method of non-linear systems with time-varying parameters. Easy tuning conditions for the controller parameter guaranteeing the stability are provided. The considered controller is based on a signal differentiator and a dynamic nullification. It turns out that the obtained results require less information on the system for the controller tuning. This approach has successfully applied on real applications such as Permanent Magnet Synchronous Motors (PMSM) and Electropneumatic System position control. These applications are published in $[14,15]$ showing the good tracking properties of this controller and the simplicity of its tuning.

In the industrial process field, digital controllers are omnipresent, and have enabled the explosion of embedded systems and networking control systems. Unlike analogical controllers, digital controllers, due to their nature, introduce discrete-time signals and discrete-time dynamics, via sample and hold devices [16]. This behavior has to be taken into account in the design of a controller as well as continuous Linear Parameter Varying (LPV) systems with a discrete time model. This difficulty has been encountered in many problems in the network control framework [17-21]. One of the solutions is to consider the sampling effect as a time varying delay with a particular shape.

Motivating by the above discussion, the main issues addressed in this work are:

(i) Proposing a new controller design procedures inspired from our previous work $[12,13]$. This controller is based on a signal differentiators and dynamic nullification and implemented in a processor based device such that the sensor data are only available at sampling instants.

(ii) By considering the discrete-time dynamics induced by the digital controller as a piecewise continuous delay, some stability results are provided. The conditions are based on Lyapunov-Krasovskii theory and polytopic transformation. The result is presented as a set of LMI (Linear Matrix Inequalities) to solve.

(iii) Proving the existence of a stabilizing derivative/controller pair for all models belonging to a special class of second order Single-Input-SingleOutput LPV systems.

(iv) Describing a simplified design procedure for the latter case.

This paper is organized as follows: The third section summarizes some of the main theoretical ideas which are shaping the model free-control presented by $[12,13]$. A time-delay approach to deal with sampling effects is proved. The fourth part of this paper is devoted to the polytopic representation of the closed loop and then to the global stability conditions synthesis. A study case of a particular class of second order non-linear LPV system is considered in section five leading to a simplified design procedure. The last part gives some conclusions and perspectives.

Notation: Throughout the article, the sets $\mathbb{N}^{*}$, $\mathbb{R}, \mathbb{R}^{n}, \mathbb{R}_{+}^{*}$ and $\mathbb{R}^{n \times n}$ denote, respectively, the set of positive integers without zero, real numbers, real positive numbers without zero, n-dimensional vectors and $n \times n$ matrices. $0_{(m \times n)}$ presents the zero matrix of dimension $m \times n$. Define $M_{((1: p) \times(1: q))}$ the $p \times q$ matrix whose entries are the $(1: p)$ and $(1: q)$ elements of the matrix $M_{((1: m) \times(1: n))}$ such that $p \leq m$ and $q \leq n$. $\operatorname{diag}(V)$ presents the $N \times N$ diagonal matrix whose entries are the $N$ elements of the vector $V . M(\alpha)$ refers to an alpha-dependant matrix. For a matrix M, symbols $M^{T}, M^{-1}, M^{(n)}, \dot{M}, \tilde{M}$ and $\hat{M}$ refer, respectively, to the transpose, the inverse, the $n^{\text {th }}$ derivative, the first derivative, an approximated value and an estimated value of M. $\tau$ is the estimator parameter. For a vector $V, V\left(s_{k}\right)$ means that the data are only available at sampling instants $s_{k} . d(t)$ is the induced time-varying delay with an upper bound $h$. * stands for the suitable expression induced by symmetry. $\mathcal{P}$ denotes a compact and convex set such that $\mathcal{P}:=\operatorname{Co}\left(P_{i}, i \in\{1 . . N\}\right):=$ $\left\{P(\sigma(t)) \mid \sum_{i=1}^{N} \mu_{i}(\sigma(t)) P_{i} ; \quad \mu_{i} \sigma(t) \in \Delta_{\mu(\sigma(t))}, \quad i \in\right.$ $\{1 . . N\}\} . \quad \mu_{i}(\sigma(t))$ are the weighting functions whose argument $\sigma(t) \in \mathbb{R}^{n_{\sigma}}$ may depend on the state or some exogenous signals. These functions should satisfy the convex sum propriety $\Delta_{\mu(\sigma(t))}:=\left\{\sum_{i=1}^{N} \mu_{i}(\sigma(t)) \sigma(t)=\right.$ $\left.1 ; \forall i \in\{1 . . N\}, 0 \leq \mu_{i}(\sigma(t)) \leq 1\right\}$.

\section{Problem formulations}

In recent years, the Linear Parameter Varying (LPV) models which represent a particular class of nonlinear systems have attracted considerable interest. Several approaches have been developed to represent, in equivalent manner, a non-linear system as LPV system [22-24]. For our study, consider the LPV system described by:

$$
\left\{\begin{array}{l}
\dot{x}_{m}(t)=A_{m}(\sigma(t)) x_{m}(t)+B_{m} u(t) \\
y(t)=C_{m} x_{m}(t)
\end{array}\right.
$$

where $x_{m}(t) \in \mathbb{R}^{\nu}$ is the state vector, $u(t) \in \mathbb{R}$ is the input vector and $y(t) \in \mathbb{R}$ is the output vector. $A_{m}(\sigma(t)) \in$ $\mathbb{R}^{\nu \times \nu}, B_{m} \in \mathbb{R}^{\nu \times 1}$ and $C_{m} \in \mathbb{R}^{1 \times \nu}$ are known matrices. $\sigma(t)$ denotes a vector of time varying parameters supposed to be non-measured but bounded in the time. Since $A_{m}(\sigma(t))$ depends on $\sigma(t)$, it can be generically describe by the polytopic form by considering the well known non-linear sector approach $[25,26]$. This transformation allows to obtain a polytopic representation of 
the state space $(1)$ in a compact and convex set $\mathcal{P}$ :

$$
\left\{\begin{array}{l}
\dot{x}_{m}(t)=\sum_{i=1}^{N} \mu_{i}(\sigma(t)) A_{m_{i}} x_{m}(t)+B_{m} u(t) \\
y(t)=C_{m} x_{m}(t)
\end{array}\right.
$$

where the integer $N$ is the number of subsystems and $A_{m_{i}} \in \mathbb{R}^{n \times n}$ are known matrices. The functions $\mu_{i}$ are the weighting functions depend on some exogenous signals. These functions verify the convex sum property $\Delta_{\mu(\sigma(t))}$ in the polytopic model domain of validity.

This paper is interested in finding the easiest method to tune dynamic output controller for this class of systems. The controller is implemented in a processor based device such that the sensor data are only available at sampling instants $s_{k}$ (Fig. 1).

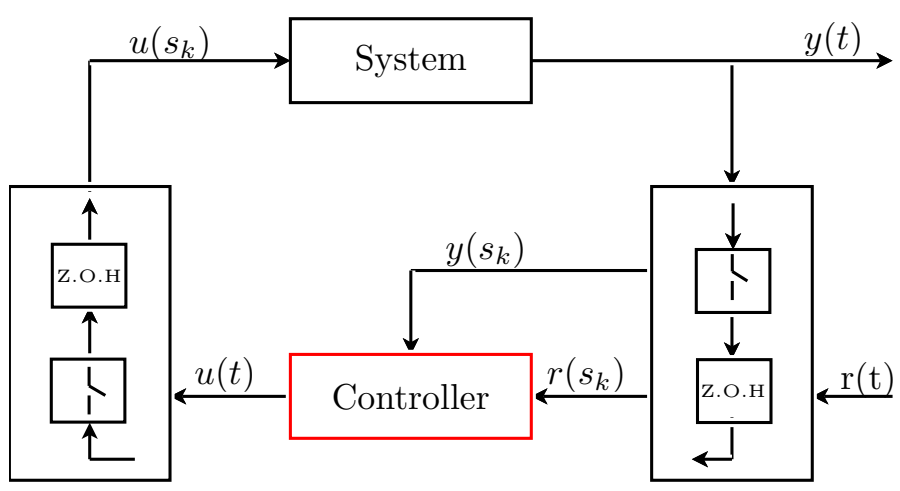

Fig. 1. Robust data sampled controller

In order to deal with the sampling effects, the LPV system (2) is then remodelled as follows:

$$
\left\{\begin{array}{l}
\dot{x}_{m}(t)=\sum_{i=1}^{N} \mu_{i}(\sigma(t)) A_{m_{i}} x_{m}(t)+B_{m} u\left(s_{k}\right) \\
y(t)=C_{m} x_{m}(t)
\end{array}\right.
$$

The controller considered in this paper is a particular case of dynamic output feedback controller. The goal being here is to tune a controller with the less information on the plant as possible i.e. by considering the largest uncertainties on the plant parameters, the less controller parameter possible and a fixed structure.

\section{The controller/derivative conception}

From the controller point of view, the dynamic of the system (3) can be replaced by the ultra-local model [8]:

$$
y^{(n)}(t)=F(t)+\alpha u\left(s_{k}\right)
$$

where $F(t)=f(y, \dot{y}, \ldots)$ is the structure function containing all-poles dynamic with eventually some distur- bances. Note that the function $F(t)$ MUST be control independent (no zero dynamics). This model considers that only the order $n \in \mathbb{N}^{*}$ of the differential equation and an approximation of the input control gain $\hat{\alpha}$ are available.

Since the idea of the controller structure is to nullify the system dynamic and then to replace it with the ideal dynamic for the closed loop, we consider the following control law introduced by [13] and represented in Fig.2:

\section{Proposition 1. Control Law}

$$
\begin{aligned}
u(t) & =\hat{u}_{n}(t)+\hat{u}_{r}(t) \\
& =-\frac{1}{\hat{\alpha}} \hat{F}(t)+\frac{1}{\hat{\alpha}}\left(-K \hat{Y}(t)+k_{0} r\left(s_{k}\right)\right)
\end{aligned}
$$

where

- $\hat{\boldsymbol{F}}(\boldsymbol{t})$ is an estimation of the "structure" function $F(t)$;

- $\tilde{\alpha}$ is an approximation of the input gain $\alpha$. Note that the choice of the parameter $\hat{\alpha}$ will be informed by the analysis of non-grouped terms in the structural function $F$;

- $\hat{\boldsymbol{Y}}(\boldsymbol{t})=\left[\begin{array}{lll}z_{0}(t) & \ldots & z_{n-1}(t)\end{array}\right]^{T}$ is a vector composed of $z_{i}(t)$ : the estimations of the successive derivatives of the system output $y^{(i)}(t)$;

- $\boldsymbol{r}\left(s_{\boldsymbol{k}}\right)$ is the reference;

- $\boldsymbol{K}=\left[k_{0}, \ldots, k_{n-1}\right]$ is a vector composed of the coefficients $k_{i}$ of the desired dynamic of the closed system given by specifications.

This controller gives a perfect closed loop only if a good estimation of the output derivatives and the function $\mathrm{F}(\mathrm{t})$ are available. There exist in the literature many techniques for the signal derivation. The simplest one is to use a filtered differentiators i.e. any stable transfer function with a stable denominator and a pure differentiators for the numerator. Other possibilities consist in the use of algebraic differentiators $[8,27]$, Luenberger unknown input observer or sliding mode observers [28-31].

In order to set a simple solution, for design purpose, we consider a simple filtered derivative approach:

$$
\left\{\begin{array}{l}
\frac{z_{0}(s)}{y(s)}=\frac{1}{\tau s+1} \\
\frac{z_{i}(s)}{y(s)}=\left(\frac{s}{\tau s+1}\right)^{i}, \quad \forall i=1 . . n-1
\end{array}\right.
$$

This estimator is causal and ensures a good estimation if its parameter $\tau$ is sufficiently smaller than the fastest dynamic of the system. It provides the successive estimations $z_{i}(t)$ of $y^{(i)}(t)$ for all $i \in\{1, \ldots, n-1\}$ where $n$ presents the system order.

The implementation of the controller in discrete time (Fig. 2) is rather simple to achieve since it is linear. However, a bad choice of the sampling period could lead to an unstable closed loop. 


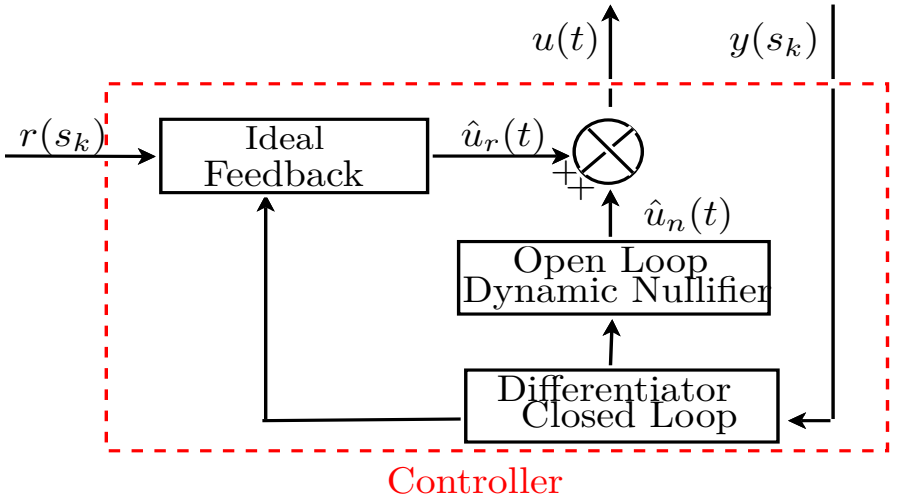

Fig. 2. Controller plant

Finally, based on the results of $[12,13]$, the sampled data controller/derivative pair composed of the control law (5) and the estimator (6) is given by the following proposition:

Proposition 2. The sampled data state representation of the controller and its derivative can be given by:

$$
\left\{\begin{array}{l}
\dot{x}_{e}(t)=A_{o} x_{e}(t)+B_{o_{1}} y\left(s_{k}\right)+B_{o_{2}} r\left(s_{k}\right) \\
u(t)=C_{o} x_{e}(t)+D_{o_{1}} y\left(s_{k}\right)+D_{o_{2}} r\left(s_{k}\right)
\end{array}\right.
$$

with $\dot{x}_{e}(t)=\left[z_{1}(t) \ldots z_{n}(t) \hat{\dot{u}}(t)\right]^{T}$ where $\frac{\hat{u}(s)}{u(s)}=\frac{1}{\tau s+1}$ and

$$
\begin{aligned}
& A_{o}=A_{e}-\frac{B_{e u}}{\hat{\hat{a}}}\left(C_{f x}+K C_{\text {eyx }}\right) \\
& B_{o_{1}}=B_{e y}-\frac{B_{e u}}{\hat{\alpha}}\left(C_{f y}+K C_{e y y}\right) \\
& B_{o_{2}}=\frac{B_{e u}}{\hat{\alpha}} k_{0} \\
& C_{o}=-\frac{1}{\hat{\hat{\alpha}}}\left(C_{f x}+K C_{\text {eyx }}\right) \\
& D_{o_{1}}=-\frac{1}{\hat{\alpha}}\left(C_{f y}+K C_{\text {eyy }}\right) \\
& D_{o_{2}}=\frac{k_{0}}{\hat{\alpha}}
\end{aligned}
$$

where the different matrices are defined by

$$
\begin{aligned}
& A_{e}=\left(\begin{array}{ccccccc}
-\frac{1}{\tau} & 0 & \ldots & \ldots & \ldots & 0 & 0 \\
-\frac{1}{\tau^{2}} & -\frac{1}{\tau} & 0 & \ldots & \ldots & 0 & 0 \\
-\frac{1}{\tau^{3}} & -\frac{1}{\tau^{2}} & -\frac{1}{\tau} & 0 & \ldots & 0 & 0 \\
\vdots & \ddots & \ddots & \ddots & \ddots & \vdots & \vdots \\
-\frac{1}{\tau^{n-1}} & -\frac{1}{\tau^{n-2}} & \ldots & \ldots & -\frac{1}{\tau} & 0 & 0 \\
-\frac{1}{\tau^{n}} & -\frac{1}{\tau^{n-1}} & \ldots & \ldots & \ldots & -\frac{1}{\tau} & 0 \\
0 & 0 & \ldots & \ldots & \ldots & 0 & -\frac{1}{\tau}
\end{array}\right) \\
& C_{f x}^{T}=\left(-\frac{1}{\tau^{n}}-\frac{1}{\tau^{n-1}} \cdots-\frac{1}{\tau}-\hat{\alpha}\right), C_{f y}=\frac{1}{\tau^{n}} \\
& C_{e u}^{T}=\left(\begin{array}{c}
0_{(1 \times n)} \\
1
\end{array}\right), C_{e y y}=\left(\begin{array}{c}
0 \\
B_{e y_{(1: n-1)}}
\end{array}\right) \\
& C_{e y x}=\left(\begin{array}{cc}
1 & 0_{(1 \times n)} \\
A_{e_{((1: n-1) \times(1: n))}} & 0
\end{array}\right), B_{e u}=\left(\begin{array}{c}
0_{(1 \times n)} \\
\frac{1}{\tau}
\end{array}\right) \\
& B_{e y}^{T}=\left(\frac{1}{\tau} \frac{1}{\tau^{2}} \ldots \frac{1}{\tau^{n}} 0\right)
\end{aligned}
$$

From this proposition, one can conclude that the proposed control law requires less information on the system for the controller tuning: only the dynamic order, an estimation of the non-linear functions bounded and an approximation of the input gain are required.

\section{Stability analyses}

Consider the sampled-data closed loop composed of the LPV system (3) and the controller/derivative pair described in Proposition 2:

$$
\left\{\begin{array}{l}
\dot{x}(t)=A(\sigma(t)) x(t)+A_{d} x\left(s_{k}\right)+B r\left(s_{k}\right) \\
y(t)=C x(t)
\end{array}\right.
$$

where $x(t)=\left[x_{m}(t) x_{e}(t)\right]^{T}$ presents the extended closed loop state and for all $\mu_{i}(\sigma(t)) \in \Delta_{\mu(\sigma(t))}$,

$$
\begin{aligned}
& A(\sigma(t))=\sum_{i=1}^{N} \mu_{i}(\sigma(t))\left(\begin{array}{cc}
A_{m_{i}} & 0 \\
0 & A_{o}
\end{array}\right) \\
& A_{d}=\left(\begin{array}{cc}
B_{m} D_{o_{1}} C_{m} & B_{m} C_{o} \\
B_{o_{1}} C_{m} & 0
\end{array}\right), B=\left(\begin{array}{c}
B_{m} D_{o_{2}} \\
B_{o_{2}}
\end{array}\right)
\end{aligned}
$$

Because the plant is not Linear time invariant, the data-sampling impact on the closed loop cannot be studied via discretization. In order to maintain the continuous nature of the system, time-delay approaches are more suitable for this case [32-34]. Modeling of continuous-time systems with digital control in the form of continuous-time systems with delayed control input was introduced by $[32,33]$ and further developed by [34]. It consists in considering the discrete-time dynamics induced by the digital controller as a piecewise continuous delay:

$$
s_{k}=t-\left(t-s_{k}\right)=t-d(t), \forall t \in\left[s_{k}, s_{k+1}\right], k \in \mathbb{N}
$$

where $s_{k}$ is the sampling instant and $d(t)=t-s_{k}$ is the induced time-varying delay satisfying $0 \leq d(t) \leq$ $h, \dot{d}(t) \leq \eta<\infty$ and $h=\tau / \kappa, \kappa \in \mathbb{R}_{+}^{*}$.

The sampled data closed loop (10) is then remodeled as a closed loop system with time-varying delay satisfying $d(t)>0$ as follow:

$$
\left\{\begin{array}{l}
\dot{x}(t)=A(\sigma(t)) x(t)+A_{d} x(t-d(t))+B r(t-d(t)) \\
x(s)=\phi(s),-h \leq s \leq 0 \\
y(t)=C x(t)
\end{array}\right.
$$

where the different matrices are defined by (11), $\phi($.$) is the initial functional such that x(s)=\phi(s) \in$ $\mathcal{L}_{2}[-h, 0] \equiv\left\{f() \mid. \int_{0}^{\infty} f^{T}(t) f(t) d t<\infty\right\}$. Here, we consider that the delay $h>d(t)>0$ is piecewise-linear with derivative $\dot{d}(t)=\eta=1$ for $t \neq s_{k}$. 
The stability of this closed loop has been studied in the linear case by [35]. The result is the following:

Theorem 1. The unforced system $\dot{x}(t)=A x(t)+$ $A_{d} x(t-d(t))+B u(t), x(s)=\phi(s)$ for $-h \leq s \leq 0$ is stable if there exist a symmetric and positive-definite common matrix $P$, common matrices $W_{1}, W_{2}, W_{3}, W_{4}$ and symmetric and positive-definite common matrices $Q, R$ and $S$ such that the following LMI holds:

$$
M=\left(\begin{array}{cccc}
M_{11} & M_{12} & M_{13} & M_{14} \\
* & M_{22} & M_{23} & M_{24} \\
* & * & M_{33} & M_{34} \\
* & * & * & M_{44}
\end{array}\right)<0
$$

where

$$
\begin{aligned}
& M_{11}=A^{T} P+P^{T} A+Q+R-W_{1}-W_{1}^{T}+h A^{T} S A \\
& M_{12}=-W_{2}+W_{1}^{T}+P A_{d}+h A^{T} S A_{d} \\
& M_{13}=-W_{3}, M_{14}=-W_{4}+W_{1}^{T} \\
& M_{22}=-(1-\eta) Q+W_{2}+W_{2}^{T}+h A_{d}^{T} S A_{d} \\
& M_{23}=W_{3}, M_{24}=W_{4}+W_{2}^{T}, M_{33}=-R, \\
& M_{34}=W_{3}^{T}, M_{44}=W_{4}+W_{4}^{T}-\frac{1}{h} S .
\end{aligned}
$$

Our goal is to extend this result to linear varying parameters (LPV) systems to guarantee the closed-loop (12) stability using some appropriate weighting matrices. In order to reduce the conservatism, time-delay dependent conditions in terms of the solutions to Linear Matrix Inequalities LMI will be developed. The following lemma is needed in the sequel.

Lemma 1. If there exist matrices $H, G(t) \in \mathcal{P}$ and $a$ symmetric and positive definite matrix $F$ with the appropriate dimensions verifying:

$$
H+\sum_{i=1}^{N} \mu_{i} \sum_{j=1}^{N} \mu_{j}\left(G_{i}\right)^{T} F G_{j}<0
$$

then,

$$
H+\left(G_{i}\right)^{T} F G_{i}<0, \forall i=1 . . n .
$$

Proof. Supposing that there exist matrices $H, G(t) \in \mathcal{P}$ and a symmetric and positive definite matrix $F$ with the appropriate dimensions verifying the condition (14). by applying the Schur Complement Theorem, this condition becomes:

$$
\sum_{i=1}^{N} \mu_{i}\left(\begin{array}{cc}
H & G_{i} \\
\left(G_{i}\right)^{T} & -F^{-1}
\end{array}\right)<0
$$

Then, we apply one again the Schur Complement The- orem:

$$
\sum_{i=1}^{N} \mu_{i}\left(H+\left(G_{i}\right)^{T} F G_{i}\right)<0
$$

The fact that $\sum_{i=1}^{N} \mu_{i}$ is bounded, the last equality implies the condition (15).

Theorem 2. The unforced system (12) is stable if there exist a symmetric and positive-definite common matrix $P$, common matrices $W_{1}, W_{2}, W_{3}, W_{4}$ and symmetric and positive-definite common matrices $Q, R$ and $S$ such that the following LMI holds:

$$
\Xi=\left(\begin{array}{cccc}
\Xi_{11} & \Xi_{12} & \Xi_{13} & \Xi_{14} \\
* & \Xi_{22} & \Xi_{23} & \Xi_{24} \\
* & * & \Xi_{33} & \Xi_{34} \\
* & * & * & \Xi_{44}
\end{array}\right)<0
$$

where for $A(\sigma(t)) \in \mathcal{P}, \forall i=1 \ldots N$ :

$$
\begin{aligned}
& \Xi_{11}=A_{i}^{T} P+P^{T} A_{i}+Q+R-W_{1}-W_{1}^{T}+h A_{i}^{T} S A_{i}, \\
& \Xi_{12}=-W_{2}+W_{1}^{T}+P A_{d}+h A_{i}^{T} S A_{d}, \\
& \Xi_{13}=-W_{3}, \Xi_{14}=-W_{4}+W_{1}^{T}, \\
& \Xi_{22}=-(1-\eta) Q+W_{2}+W_{2}^{T}+h A_{d}^{T} S A_{d}, \\
& \Xi_{23}=W_{3}, \Xi_{24}=W_{4}+W_{2}^{T}, \Xi_{33}=-R, \\
& \Xi_{34}=W_{3}^{T}, \Xi_{44}=W_{4}+W_{4}^{T}-\frac{1}{h} S .
\end{aligned}
$$

Proof. Consider the proof of the Theorem 1 developed by [35] with the following Lyapunov-Krasovskii functional:

$$
V(x(t))=V_{1}(x(t))+V_{2}(x(t))+V_{3}(x(t))+V_{4}(x(t))
$$

where

$$
\begin{aligned}
& V_{1}(x(t))=x^{T}(t) P x(t) \\
& V_{2}(x(t))=\int_{t-d(t)}^{t} x^{T}(s) Q x(s) d s \\
& V_{3}(x(t))=\int_{t-h}^{t} x^{T}(s) R x(s) d s \\
& V_{4}(x(t))=\int_{-h}^{0} \int_{t+\theta}^{t} \dot{x}^{T}(s) S \dot{x}(s) d s d \theta
\end{aligned}
$$

with $P>0, Q>0, R>0$ and $S>0$. Since the state matrix parameters are supposed time-varying such that $A(t)=\sum_{i=1}^{N} \mu_{i}(\sigma(t)) A_{i}, M_{11}$ and $M_{12}$ of the Theorem 1 became:

$\begin{aligned} M_{11}= & \sum_{i=1}^{N} \mu_{i}(\sigma(t)) A_{i}^{T} P+\sum_{i=1}^{N} \mu_{i}(\sigma(t)) P^{T} A_{i}+Q+R-W_{1} \\ & -W_{1}^{T}+h \sum_{i=1}^{N} \mu_{i}(\sigma(t)) \sum_{j=1}^{N} \mu_{j}(\sigma(t)) A_{i}^{T} S A_{j} \\ M_{12}= & -W_{2}+W_{1}^{T}+P A_{d}+h \sum_{i=1}^{N} \mu_{i}(\sigma(t)) A_{i}^{T} S A_{d}\end{aligned}$

Using Lemma 1 and relations given in the proof of [35], one gets:

$$
M=\sum_{i=1}^{N} \mu_{i}(\sigma(t)) \Xi<0
$$


were $\sum_{i=1}^{N} \mu_{i}(\sigma(t))$ is bounded and implies $\Xi<0$.

\section{Study case: second order LPV systems}

The stability of the closed loop with time-varying delay for a given system was proven in the last section. Since the model free control is sensitive to the presence of invariant zeros [36], the following class of second order single input single output linear system with time varying parameters and delay will be considered in the rest of this paper:

$$
\begin{aligned}
& y^{(2)}(t)=-a_{0}(t) y(t)-a_{1}(t) \dot{y}(t)+\alpha u(t-d(t)), \\
& \left|a_{i}(t)\right|<\bar{a}_{i} \quad \forall i=0,1
\end{aligned}
$$

where $y(t) \in \mathbb{R}$ is the system output, $u(t-d(t)) \in \mathbb{R}$ is the control input, $\alpha$ is the input gain and $a_{i} \in \mathbb{R}$ are scalar time varying unknown parameters that their absolute value is bounded by $\bar{a}_{i}, \forall i=0,1$ i.e. $a_{0}(t) \in\left[-\bar{a}_{0}+\bar{a}_{0}\right]$ and $a_{1}(t) \in\left[-\bar{a}_{1}+\bar{a}_{1}\right]$.

By choosing $x_{m}(t)=[y(t) \dot{y}(t)]^{T}$ as a state space vector, the closed loop system with time-varying delay composed of the second order system (18) and the controller/derivative pair (7) can be represented by the state space form (12) as follows:

$$
\left\{\begin{array}{l}
\dot{x}(t)=A(\sigma(t)) x(t)+A_{d} x(t-d(t))+B r(t-d(t)) \\
y(t)=C x(t)
\end{array}\right.
$$

where the different matrices are defined by (11) with (8), (9) and:

$$
\begin{aligned}
& A_{m_{1}}=\left(\begin{array}{cc}
0 & 1 \\
+\bar{a}_{0} & +\bar{a}_{1}
\end{array}\right), A_{m_{2}}=\left(\begin{array}{cc}
0 & 1 \\
+\bar{a}_{0} & -\bar{a}_{1}
\end{array}\right) \\
& A_{m_{3}}=\left(\begin{array}{cc}
0 & 1 \\
-\bar{a}_{0} & +\bar{a}_{1}
\end{array}\right), A_{m_{4}}=\left(\begin{array}{cc}
0 & 1 \\
-\bar{a}_{0} & -\bar{a}_{1}
\end{array}\right) \\
& B_{m}=\left(\begin{array}{l}
0 \\
\alpha
\end{array}\right) \quad, C_{m}=\left(\begin{array}{ll}
1 & 0
\end{array}\right)
\end{aligned}
$$

\subsection{Tuning Method}

The first result brings out the general nature of the controller by ensuring that if there exist a solution to the stabilization problem for this class of second order system with a some non zero $\overline{a_{i}}$, then there is a solution for any systems of this class i.e. any $\overline{a_{i}}$. In addition, this result is constructive since it allows to compute the controller parameters. These results are formalized in the following theorem:

Theorem 3. If the closed loop composed of the system

$$
\Sigma_{a}: y^{(2)}(t)=-a_{0}(t) y(t)-a_{1}(t) \dot{y}(t)+\alpha u\left(t-d_{x}(t)\right)
$$

and the controller/derivative pair

$\left\{\begin{array}{l}\dot{x}_{e}(t)=A_{o}\left(\tau_{x}\right) x_{e}(t)+B_{o_{1}}\left(\tau_{x}\right) y\left(t-d_{x}(t)\right)+B_{o_{2}}\left(\tau_{x}\right) r\left(t-d_{x}(t)\right) \\ u(t)=C_{o}\left(\tau_{x}\right) x_{e}(t)+D_{o_{1}}\left(\tau_{x}\right) y\left(t-d_{x}(t)\right)+D_{o_{2}}\left(\tau_{x}\right) r\left(t-d_{x}(t)\right.\end{array}\right.$ where the different matrices are given by (8), is asymptotically stable for all continuous and bounded function

$$
\begin{gathered}
a_{i}(.): \mathbb{R}^{2} \mapsto\left[-\bar{a}_{i}, \bar{a}_{i}\right], i \in\{0,1\} \\
0<d_{x}(t)<h_{x}
\end{gathered}
$$

Then, there exists a stabilizer controller/derivative for all system

$$
\Sigma_{b}: z^{(2)}(t)=-b_{0}(t) z(t)-b_{1}(t) \dot{z}(t)+\beta v\left(t-d_{z}(t)\right)
$$

with

$$
\begin{gathered}
b_{i}(.): \mathbb{R}^{2} \mapsto\left[-\gamma^{2-i} \bar{a}_{i}, \gamma^{2-i} \bar{a}_{i}\right], i \in\{0,1\} \\
\beta=\gamma^{2} \alpha \\
0<d_{z}(t)<h_{z}
\end{gathered}
$$

The new controller/derivative pair of the system $\Sigma_{b}$ is then deduced from the one of the system $\Sigma_{a}$ as follows:

$\left\{\begin{array}{l}\dot{z}_{e}(t)=A_{o}\left(\tau_{z}\right) z_{e}(t)+B_{o_{1}}\left(\tau_{z}\right) z(t-d(t))+B_{o_{2}}\left(\tau_{z}\right) w(t-d(t)) \\ v(t)=C_{o}\left(\tau_{z}\right) z_{e}(t)+D_{o_{1}}\left(\tau_{z}\right) z(t-d(t))+D_{o_{2}}\left(\tau_{z}\right) w(t-d(t))\end{array}\right.$

such that

$$
\begin{aligned}
& A_{o}\left(\tau_{z}\right)=\gamma T_{2} A_{o}\left(\tau_{x}\right) T_{2}^{-1} \\
& B_{o_{1}}\left(\tau_{z}\right)=\gamma T_{2} B_{o_{1}}\left(\tau_{x}\right) \\
& B_{o_{2}}\left(\tau_{z}\right)=\gamma T_{2} B_{o_{2}}\left(\tau_{x}\right) \\
& C_{o}\left(\tau_{z}\right)=C_{o}\left(\tau_{x}\right) T_{2}^{-1} \\
& D_{o_{1}}\left(\tau_{z}\right)=D_{o_{1}}\left(\tau_{x}\right) \\
& D_{o_{2}}\left(\tau_{z}\right)=D_{o_{2}}\left(\tau_{x}\right) \\
& K_{z}=\gamma^{n} K_{x} T_{1}^{-1} \\
& w(t)=r(\gamma t)
\end{aligned}
$$

with $0<d(t)<h=\max \left(h_{x}, h_{z}\right)$ and

$$
\begin{aligned}
& \gamma=\tau_{x} / \tau_{z} \\
& T_{1}=\operatorname{diag}(1, \gamma) \\
& T_{2}=\operatorname{diag}(1, \gamma, 1)
\end{aligned}
$$

Proof. Supposing that the system closed loop composed of the SISO system given by (20) and the controller/derivative given by (21) is stable. In this case, the state space representation (12) becomes:

$$
\begin{aligned}
\frac{d}{d t}\left(\begin{array}{c}
x_{m}(t) \\
x_{e}(t)
\end{array}\right) & =\left(\begin{array}{cc}
A_{m}(\sigma(t)) & 0 \\
0 & A_{o}\left(\tau_{x}\right)
\end{array}\right)\left(\begin{array}{c}
x_{m}(t) \\
x_{e}(t)
\end{array}\right) \\
& +\left(\begin{array}{cc}
B_{m} D_{o_{1}}\left(\tau_{x}\right) C_{m} & B_{m} C_{o}\left(\tau_{x}\right) \\
B_{o_{1}}\left(\tau_{x}\right) C_{m} & 0
\end{array}\right)\left(\begin{array}{c}
x_{m}\left(t-d_{x}(t)\right) \\
x_{e}\left(t-d_{x}(t)\right)
\end{array}\right) \\
& +\left(\begin{array}{c}
B_{m} D_{o_{2}}\left(\tau_{x}\right) \\
B_{o_{2}}\left(\tau_{x}\right)
\end{array}\right) r\left(t-d_{x}(t)\right)
\end{aligned}
$$

where the matrices are described by (8) and (9). Then, 
this state space can be written as:

$\left\{\begin{aligned} \frac{d}{d t} X(t) & =\mathcal{A}\left(\sigma(t), \tau_{x}\right) X(t)+\mathcal{A}_{d}\left(\tau_{x}\right) X\left(t-d_{x}(t)\right)+\mathcal{B}\left(\tau_{x}\right) r\left(t-d_{x}(t)\right) \\ Y(t) & =C X(t)\end{aligned}\right.$

Consider the following variable change: $w(t-$ $\left.d_{z}(t)\right)=r\left(\gamma t-d_{x}(\gamma t)\right)$ where $0<d_{z}(t)<h_{z}, \gamma \in \mathcal{R}_{+}^{*}$ and $Z(t)=Q X(\gamma t)$, such that $Q=\operatorname{diag}\left(T_{1}, T_{2}\right), T_{1}=$ $\operatorname{diag}(1, \gamma)$ and $T_{2}=\operatorname{diag}(1, \gamma, 1)$. In this case, the closed loop (29) becomes:

$$
\frac{d}{d t} Z(t)=\frac{d}{d t}(Q X(\gamma t))=Q \frac{d}{d t}(X(\gamma t))=Q \gamma \frac{d X}{d t}(\gamma t)
$$

i.e.

$$
\begin{aligned}
& \frac{d}{d t} Z(t)=\quad Q \gamma\left(\mathcal{A}\left(\sigma(\gamma t), \tau_{x}\right) X(\gamma t)+\mathcal{A}_{d}\left(\tau_{x}\right) X\left(\gamma t-d_{x}(\gamma t)\right)\right. \\
& \left.+\mathcal{B}\left(\tau_{x}\right) r\left(\gamma t-d_{x}(\gamma t)\right)\right) \\
& \begin{array}{c}
=Q \gamma \mathcal{A}\left(\sigma(\gamma t), \tau_{x}\right) Q^{-1} Z(t)+Q \gamma \mathcal{A}_{d}\left(\tau_{x}\right) Q^{-1} Z\left(t-d_{z}(t)\right) \\
+\gamma Q \mathcal{B}\left(\tau_{x}\right) w\left(t-d_{z}(\gamma t)\right)
\end{array} \\
& =\quad \gamma\left(\begin{array}{cc}
T_{1} A_{m}(\sigma(t)) T_{1}^{-1} & 0 \\
0 & T_{2} A_{o}\left(\tau_{x}\right) T_{2}^{-1}
\end{array}\right) Z(t) \\
& +\gamma\left(\begin{array}{cc}
T_{1}\left(B_{m} D_{o_{1}}\left(\tau_{x}\right) C_{m}\right) T_{1}^{-1} & T_{1} B_{m} C_{o}\left(\tau_{x}\right) T_{2}^{-1} \\
T_{2} B_{o_{1}}\left(\tau_{x}\right) C_{m} T_{1}^{-1} & 0
\end{array}\right) Z\left(t-d_{z}(t)\right) \\
& +\gamma\left(\begin{array}{c}
T_{1} B_{m} D_{o_{2}}\left(\tau_{x}\right) \\
T_{2} B_{o_{2}}\left(\tau_{x}\right)
\end{array}\right) w\left(t-d_{z}(t)\right)
\end{aligned}
$$

Since the variable change is linear, this latter equation is asymptotically stable for all continuous and bounded function described by (22). As a matter of fact, the system

$$
\begin{aligned}
& z^{(2)}(t)=-\gamma^{2} a_{0}(t) z(t)-\gamma a_{1}(t) \dot{z}(t)+\gamma^{2} \alpha v\left(t-d_{z}(t)\right) \\
& \left|a_{i}(t)\right| \leq \bar{a}_{i}, \quad \forall i \in\{0,1\}
\end{aligned}
$$

can be stabilized by the controller/derivative pair (25) described by the matrices given by (26) with (27). Finally, by considering the variable change $b_{i}=\gamma^{2-i} a_{i}$ and $\beta=\gamma^{2} \alpha$, one can assume that this system closed loop is stable for all continuous and bounded function described by (24).

Considering the results of the previous Theorem, the second result is devoted to propose a simplified method to compute the controller parameter $\tau$ for the class systems defined by equation (18). The Fig.3 presents the stability area of the closed loop system by considering the landmark $\left(\tau_{x}^{2} \bar{a}_{0}, \tau_{x} \bar{a}_{1}\right)$. This domain has been computed online by applying the Theorem 2 with the following parameters:

- a null reference : $r(t)=0$;

- $K_{x}=\left[\begin{array}{ll}10^{-4} & 2.210^{-2}\end{array}\right]$ i.e. the desired dynamic is given by: $y_{r}^{(2)}=-10^{-4} y_{r}-2.210^{-2} \dot{y}_{r}$;

- $\tau_{x}=0.01$;

- $\tilde{\alpha}=\alpha$ and then without loss of generality $\alpha=1$;

- $N=2^{2}$.

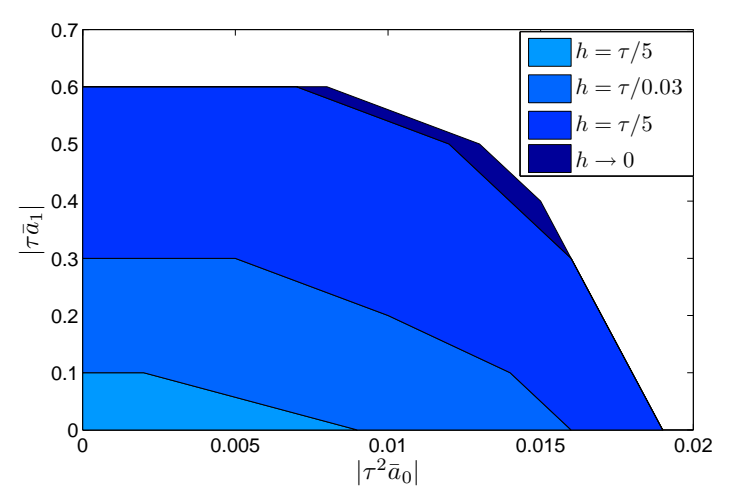

Fig. 3. Stability set of the closed loop (19) for $\tau_{x}=0.01, \alpha=1$, $K_{x}=\left[10^{-4} 2.210^{-2}\right]$ and different delays $h_{x}$

According to the values of $a_{0}$ and $a_{1}$, we can see in Fig. 3 that the stability region of the system (19) is related to the time delay $h$ and more precisely to the ration between $\tau$ and the sampling period. Yet, from this figure and the results given in $[12,13]$, we note that this stability domain of the same LPV system with a time-varying delay is smaller than that of the continuous case $(h \rightarrow 0)$.

From this Figure, it is possible to tune the parameter $\tau$ ensuring the stability of all system of the class (18). For example, consider the following system:

$$
z^{(2)}(t)=-b_{0}(t) z(t)-b_{1}(t) \dot{z}(t)+\beta v\left(t-d_{z}(t)\right)
$$

with $b_{0} \in[-200,200], b_{1} \in[-70,70]$ and $0<d_{z}(t)<h_{z}$.

From the Fig.3, one can see that a value of $\tau_{z}=$ 0.005 places the closed loop in the stable area of $h_{x}=$ $\tau_{x} / 5=0.002$. This implies from Theorem 3 that the controller (25) defined by (26) and (27) where $\gamma=$ $\tau_{x} / \tau_{z}=0.01 / 0.005$ stabilizes the system. It should be stressed that applying the Theorem 3 changes the rapidity of the desired dynamic (but the shape of the response will be the same) which will then be given by the differential equation: $z_{r}^{(2)}=-410^{-4} z_{r}-4.410^{-2} \dot{z}_{r}$ i.e $K_{z}=\gamma^{2} K_{x} \operatorname{diag}(1,1 / \gamma)$. Nevertheless, this theorem does not predict the tracking quality of the desired dynamic.

One can conclude from this example and Fig.3 that for all second order system with bounded parameters and described by the differential equation (18), there exists a stabilizing $\tau$ where the corresponding controller is described in Proposition 5 and its parameter is tuned via the following proposition:

Proposition 3. For all linear second order SISO system (18) with bounded time varying parameters $\left|a_{i}(t)\right| \leq$ $a \forall i \in\{0,1\}$, there exists a controller defined by (25) with (26) and (27) stabilizing the sampled data closed loop (3). This controller is tuned by choosing a value of 
$\tau$ and $h$ such that

$$
\begin{aligned}
& \tau^{2-i} * a<10^{-2} \quad \forall i=0,1 \\
& h=\tau / 5 \\
& K=\left[\begin{array}{ll}
10^{-4} / \tau^{2} & 0.022 / \tau
\end{array}\right]
\end{aligned}
$$

Proof. The proof of this proposition follows from the numerical computation of the stability set of the second order systems and the use of Theorem 3 .

Remark 1. Since the stability is preserved even for low value of the parameter $\tau$, it is possible to accelerate the desired dynamic response without affecting the stability. If the shape of the response is not satisfying, one must compute the stability area related to this new dynamic as was the case in the previous example (Fig.3).

To conclude, one can assume that only the system bounded and the input gain value $\alpha$ are required for the stability study of the second order class of systems described by (18) since there exists always a value of $\tau$ ensuring the sampled data closed loop stability.

\subsection{Robustness tests}

Another important point concerns the approximation of the parameter $\alpha$. By applying the stability theorem of the polytopic systems, the Fig. 4 depicts the set for which the closed loop stability is guaranteed for $\tilde{\alpha} / \alpha \in\{3,1,0.8\}$ where $\hat{\alpha}$ is an approximation of $\alpha$. One can clearly see that when large uncertainty on the knowledge of the input gain $\alpha$ occurs, the closed loop stability can be preserved by considering smaller value of $\tau$.

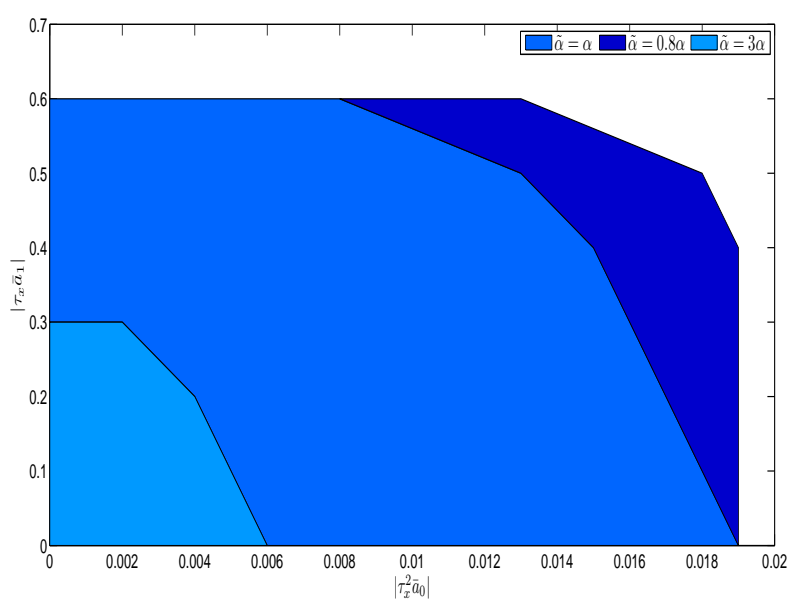

Fig. 4. Stability set of the second order system (19) with $\tau=0.01$ and $K=\left[10^{-4} 2.210^{-2}\right]$ for different approximation of the input gain $\alpha$ and a time-delay $h=\frac{\tau}{5}$

\subsection{Example: Closed loop simulation}

Consider a second order LPV system defined by the dynamic (18) such that $a_{0}(t)=20 \sin (t)$ and $a_{1}(t)=$ $-40 \sin (t)$ such that $\bar{a}_{0}=20$ and $\bar{a}_{1}=40$ are the system parameters bounded. By applying the stability Theorem 2 using SeDuMi [37] as solver and YALMIP [38] as parser in MATLAB, a feasible solution is provided.

A simulation of this system is given to verify the analysis results in terms of stability and performance. Fig. 5 shows the regulation results of states $y$ and $\dot{y}$. All the states successfully converge to zero, which means that the proposed scheme solves the regulation problem even in the case when the system has unknown parameters and its states are not measurable. The performance of the proposed controller/derivative pair is shown in Fig. 6. This control law affects how fast the estimated states catch up with original states. Finally, the control input $\mathrm{u}$ converges to zero, as shown in Fig. 7.

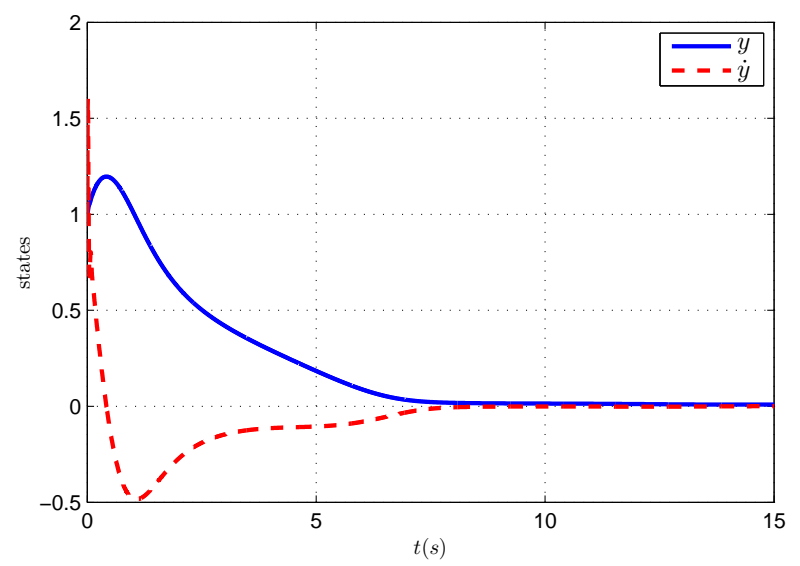

Fig. 5. Response of the system states

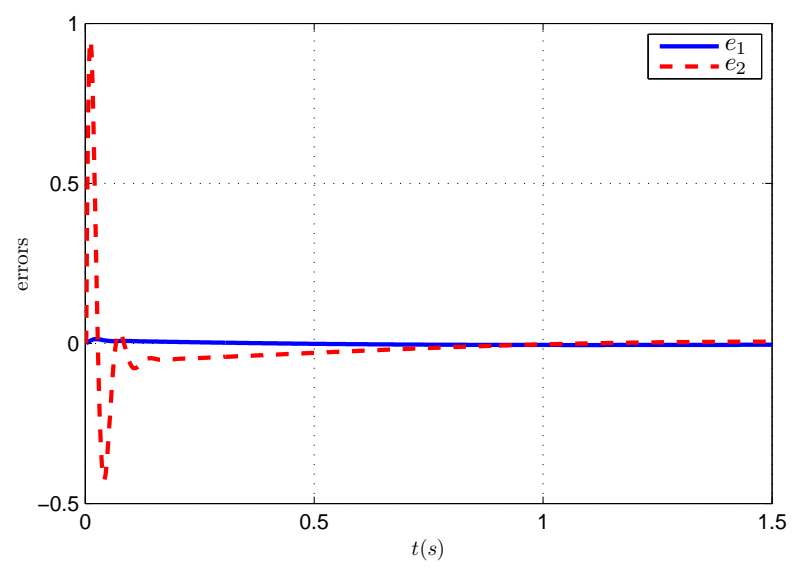

Fig. 6. States estimation errors 


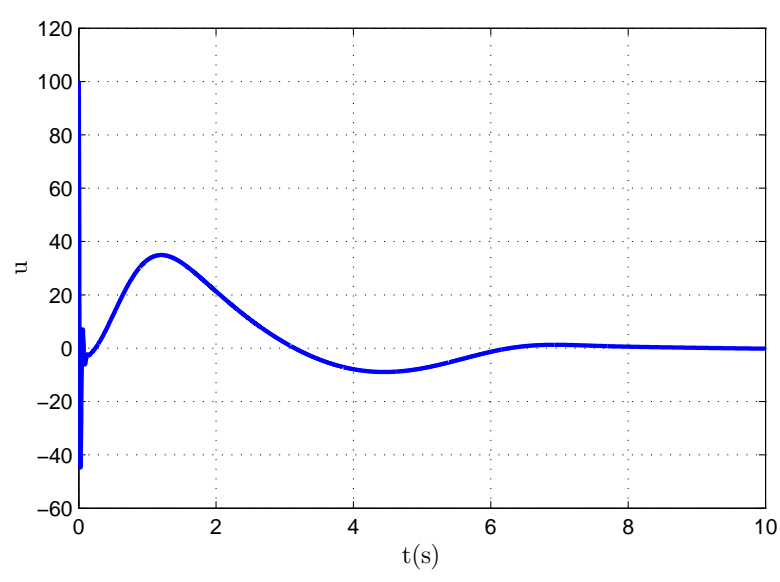

Fig. 7. Control input

\section{6 conclusions}

In contrast of observer based controllers, this paper proposed a dynamic output controller allowing the stabilisation of a class of LPV systems with non-measurable parameters. This controller is implemented in a processor based device such that the sensor data are only available at sampling instants. Based on our previous results $[12,13]$ and by considering the discrete-time dynamics induced by the digital controller as a piecewise continuous delay, some stability results are provided. The usefulness and robustness of the approach is shown trough some numerical examples via the study case of the second order SISO systems. Our future work will deal with extended the class of system (multi-input multi-output, zero dynamics), guaranteed performances and noise rejection of the sampled data systems.

\section{References \\ References}

[1] Åström, K., and ägglund, T. H., 2006. Advanced PID Control. The Instrumentation, Systems, and Automation Society; Research Triangle Park, NC 27709.

[2] Khodabakhshian, A., and Edrisi, M., 2008. "A new robust $\{$ PID $\}$ load frequency controller". Control Engineering Practice, 16(9), pp. 1069 - 1080.

[3] Huang, D., and Nguang, S. K., 2007. "Static output feedback controller design for fuzzy systems: An ilmi approach". Inf. Sci., 177(14), pp. 3005-3015.

[4] Mansouri, B., Manamanni, N., Guelton, K., Kruszewski, A., and Guerra, T. M., 2009. "Output feedback lmi tracking control conditions with $h_{\infty}$; criterion for uncertain and disturbed t-s models". Inf. Sci., 179(4), pp. 446-457.

[5] Sato, M., 2011. "Gain-scheduled output-feedback controllers depending solely on scheduling parameters via parameter-dependent lyapunov functions". Automatica, 12, pp. 2786-2790.

[6] Tanaka, K., Ikeda, T., and Wang, H., 1998.
"Fuzzy regulators and fuzzy observers: relaxed stability conditions and lmi-based designs". IEEE Transactions on Fuzzy Systems, 6(2), pp. 250-265.

[7] Sala, A., Guerra, T. M., and Babuška, R., 2005. "Perspectives of fuzzy systems and control". Fuzzy Sets and Systems, 156(3), pp. $432-444$.

[8] Join, C., Masse, J., and Fliess, M., 2008. "Etude préliminaire d'une commande sans modèle pour papillon de moteur ..... A model-free control for an engine throttle: a preliminary study". Journal Européen des Systèmes Automatisés, 42(2-3), pp. 337-354.

[9] Abouaissa, H., Fliess, M., Iordanova, V., and Join, C., 2011. "First steps towards a model-free control of a freeway traffic flow-Prolégomènes à une régulation sans modèle du trafic autoroutier". In Conférence Méditerranée sur l'Ingénierie Sûre des Systèmes Complexes.

[10] Gédouin, P., Delaleau, E., Bourgeot, J., Join, C., Arbab-Chirani, S., and Calloch, S., 2011. Experimental comparison of classical pid and model-free control: position control of a shape memory alloy active spring. Elsevier, May.

[11] D'Andréa-Novel, B., Boussard, C., Fliess, M., El Hamzaoui, O., Mounier, H., and Steux, B., 2010. "Commande sans modèle de la vitesse longitudinale d'un véhicule électrique". In Sixième Conférence Internationale Francophone d'Automatique.

[12] Maalej, S., Kruszewki, A., and Belkoura, L., 2013. "Derivative based control for ltv system with unknown parameters". European Control Conference.

[13] Maalej, S., Kruszewski, A., and Belkoura, L., 2016. "Robust Control for Continuous LPV System with Restricted-Model-Based Control". Circuits, Systems, and Signal Processing.

[14] Maalej, S., Kruszewski, A., Delpoux, R., and Belkoura, L., 2014. "Derivative based control for lpv system with unknown parameters: An application on a permanent magnet synchronous motors". In International Multi-Conference on Systems, Signals Devices, pp. 1-6.

[15] Harchay, M., Louhichi, B., Maalej, S., and Smaoui, M., 2015. "On the model-free control of an electropneumatic system". In International conference on Sciences and Techniques of Automatic control and computer engineering.

[16] Åström, K., and Wittenmark, B., 1984. Computer controlled systems: theory and design. PrenticeHall.

[17] Fridman, E., Seuret, A., and Richard, J.-P., 2004. "Robust sampled-data stabilization of linear systems: an input delay approach". Automatica, 40(8), pp. $1441-1446$.

[18] Fridman, E., 2010. "A refined input delay approach to sampled-data control". Automatica, 46 (2), pp. $421-427$.

[19] Seuret, A., 2009. "Stability analysis for sampleddata systems with a time-varying period". IEEE 
Conference on Decision and Control, pp. 8130 8135.

[20] Hetel, L., Kruszewski, A., Perruquetti, W., and Richard, J. P., 2011. "Discrete and intersample analysis of systems with aperiodic sampling". IEEE Transactions on Automatic Control, 56(7), pp. 1696-1701.

[21] Fiter, C., Hetel, L., Perruquetti, W., and Richard, J.-P., 2012. "A state dependent sampling for linear state feedback". Automatica, 48(8), pp. $1860-$ 1867.

[22] Hecker, S., and Varga, A., 2004. "Generalized lftbased representation of parametric uncertain models". European Journal of Control, 10(4), pp. 326337.

[23] Marcos, A., and Balas, J., 2004. "Development of linear-parameter-varying models for aircraft". J.

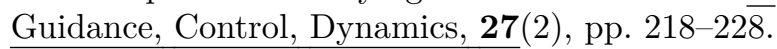

[24] Tan, W., 1997. "Applications of linear parametervarying control theory". PhD thesis, Dept. of Mechanical Engineering, University of California at Berkeley, Berkeley.

[25] S.Kawamoto, Tada, K., Ishigame, A., and Taniguchi, T., 1992. "An approach to stability analysis of second order fuzzy systems". In IEEE International Conference on Fuzzy Systems, pp. 1427-1434.

[26] Tanaka, K., Hori, T., and Wang, H., 2001. "A fuzzy lyapunov approach to fuzzy control system design". In Proceedings of the American Control Conference, Vol. 6, pp. 4790-4795.

[27] Mboup, M., 2009. "Parameter estimation for signals described by differential equations". Applicable Analysis, 88(1), pp. $29-52$.

[28] Slotine, J., 1984. "Sliding controller design for nonlinear systems". International Journal of Control, $\underline{\mathbf{4 0}}(2)$, pp. $421-434$.

[29] Nounou, M. N., Nounou, H. N., and Mahmoud, M. S., 2007. "Robust adaptive sliding-mode control for continuous time-delay systems". IMA Journal of Mathematical Control and Information, 24(3), pp. 299-313.

[30] Yan, S., and Sun, Z., 2010. "Study on separation principles for t-s fuzzy system with switching controller and switching observer". Neurocomputing, 73(13-15), pp. 2431-2438.

[31] Feng, Y., and Wang, L., 2010. "Terminal sliding mode observer based parameter estimation method for permanent magnet synchronous motor control system". International Workshop on Variable Structure Systems, pp. 184-189.

[32] Fridman, E., Micheev, Y., and Sobolev, V., 1988. "Asymptotic analysis of digital control systems". Automation and remote control, 49, pp. 11751180 .

[33] Åström, K., and Wittenmark, B., 1995. Adaptive Control. Addison-Wesley.

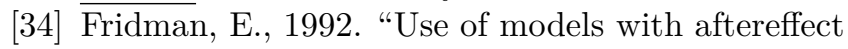

in the problem of design of optimal digital control". Automation and remote control, 53(10), pp. 15231528.

[35] Boukas, E., 2008. "Free-weighting matrices delaydependent stabilization for systems with timevarying delays". ICIC Express Letters, 2(2), pp. $167-173$.

[36] Fliess, M., and Join, C., 2013. "Model-free control". International Journal of Control, 86(12), pp. 22282252.

[37] Sturm, J. F., 1999. "Using sedumi 1.02, a matlab toolbox for optimization over symmetric cones". Optimization Methods and Software, 11 (14), pp. 625-653.

[38] Lofberg, J., 2004. "Yalmip : a toolbox for modeling and optimization in matlab". In IEEE International Symposium on Computer Aided Control Systems Design, pp. 284-289. 